\title{
A GRAPH BASED APPROACH FOR EFFECTIVE INFLUENCER MARKETING
}

\author{
Salman Ansari, Muhammad Ahsan Tahir and Shahwaiz Bukhari \\ Techlets Pvt. Ltd.
}

\begin{abstract}
We present a novel graph-based approach to find the optimal set of influencers from a large pool of influencers. The goal is to select minimum number of influencers that can reach the desired audience. In order to find such a set, one has to compute the reach of all possible combinations of available influencers resulting in complexity of order $O\left(2^{n}\right)$. Our proposed greedy approach selects the pair of influencers that results in highest reach at every iteration reducing the complexity to $O\left(n^{2}\right)$. Our work is complimented with analysis of 550 Instagram influencers and over 100,000 post. After the analysis, we concluded that influencers who prefer quality over quantity receives better engagement. Influencers sharing 3 posts per week and posts with caption length of over 500 characters relatively received better engagement numbers.
\end{abstract}

\section{KEYWORDS}

Graph Analytics, Influencer Marketing, Social Media Influencer, Greedy Algorithm

\section{INTRODUCTION}

With 3G/4G telecom services now common and offered at among the world's most affordable rates in Pakistan, 67 million locals have mobile broadband internet access. According to a Gallup \& Gillani poll, $48 \%$ of internet users consume online content daily[1]. One content producer category is the "Social/Digital Influencer" who are followed by 'fans' numbering from a few thousands (micro-influencers) to millions (celebrities). Their digital content, whether pictures, videos and live streams, are delivered on digital and social channels - Instagram, Facebook, YouTube, Beigo, TikTok - and cover a wide range of genres from lifestyle, fashion, health to entertainment and comedy.Brands can use social and digital influencers to launch and manage effective organic marketing campaigns. Pakistan's SMEs, comprising $98 \%+$ of all companies, actively use influencer marketing as a cost-effective strategy to engage and sell in/directly to Pakistan's online population[2], an estimated "trade" value at PKR 5 billion*/year[3]. For sellers, influencers represent an impactful and efficient outreach channel as they affect purchase behaviors and choices, brand perceptions and even the national narratives of $\sim 30 \%$ of Pakistan's population[4].

A key challenge is to optimize influencer marketing budgets by cost-efficiently identifying the most optimal set of influencers who can effectively reach desired audiences, from prospects, buyers to promoters. To only consider an influencer's number of followers is misleading as there is an underlying overlap between the followers of influencers who have contextual similarities that is two influencers with the same city, age group, same genre/category and content themes are likely to appeal to the same people following them both. To solve this overlap problem, we are focusing on one of the most influencer-driven social networks, Instagram. We propose a Greedy Algorithm to rationalize the minimum number of influencers to attain the best reach. 
Our proposed solution is supported by analyzing500+ influencer profiles and over 100,000 Instagram posts to establish trends and benchmarks for influencers. For example; the average post's engagement is $3.28 \%$ of total followers. Based on our findings those influencers who consistently had a higher engagement rate, appeared to emphasize on quality over quantity, as posts had detailed and meaningful caption length swith frequency of up to three posts/week. The key constraint to our approach is to have a comprehensive list of followers of all the influencers as overlap cannot be found between the influencers without the complete list of all the users following the influencers.

\section{RELATED WORK}

Influencer marketing is described as the "art and science of engaging people who are influential online to share brand messages with their audiences in the form of sponsored content" [5].Social media influencers who are responsible for influencer marketing are the third-party endorser responsible for shaping attitude of the audience towards the brands through videos, blogs and social media [6].

To solve our defined problem, we explored graph-based solutions as Influencers-Followers relationships that can best be explained in graphical representation with nodes as Influences/Followers and edges as interactions between them. We examined several different techniques and tried to model our problem on them. Bhamaikar and Rao [7] worked on identifying cliques using degree and connectivity constraints, we tried to model our problem and modify their approach to create and analyze cliques of followers to find the true reach of all influencers but realized that clique detection was not the right representation of the problem we were working to solve. Leskovec, Lang, Dasgupta, Mahoney [8]employed approximation algorithms to identify community structures in large social networks, we intended to employ their approach to estimate true reach of Influencers by identify communities of followers. Leskovec, Backstrom and Kleinberg [9] suggested to monitor information flow using content of the information like hash tags or memes. Sawhney, Prasetio and Paul [10] combined graph structure and semantic understanding of the text for community detection. We worked to identify overlapping followers by monitoring the content they post on their profiles but it is too much of an overhead to monitor the content of all followers of the influencers.

To the best of our knowledge and research, we were unable to find any body of work directly related to our problem. We therefore propose novel approach to solve the problem of finding effective influencers by introducing the "Greedy Algorithm".

\section{KEY DEFINITIONS:}

\subsection{Influencers:}

Instagram users with a substantial following, engagement rates, working with brands or creating their own content for the purpose of advertisement, promotions and outreach.

\subsection{Overlapping Followers:}

The followers of an influencer who also follows other influencers. If user ' $\mathrm{A}$ ' is following two influencers IA and IB than ' $A$ ' is considered the overlapped follower of IA and IB. 


\subsection{Reach:}

The maximum number of users who can be reached with a selected influencer. For any influencer, reach is the total number of its followers.

\subsection{True reach of influencers:}

The number of distinct users who can be reached with selected influencers. For two influencers IA and IB,it is calculated as:

$$
\text { TrueReachofIAandIB }=(\text { ReachofIA }+ \text { ReachofIB })-\text { OverlappingFollowers }
$$

\subsection{Engagement:}

The total activity received on any post, defined as:

$$
\text { Engagement }=\text { SumofLikes }+ \text { SumofComments }
$$

\subsection{Influencer Node:}

The nodes in the graph representing influencers.

\subsection{Follower Node:}

The nodes in the graph representing followers.

\section{Problem Statement}

Marketing agencies can select from their in-house directory of influencers talent to be allocate to a marketing campaign. As one Instagram user can follow several users on the platform, there is a high probability that there will be overlapping of followers between the influencers (a follower following more than one influencer), hence the actual reach of the selected influencers will be far less than the sum of total number of their followers.

Every brand wants to optimize marketing spend. They want to continuously improve reach metrics by using the right combination of influencers to maximize reach using minimal budget spend.

Our sample dataset [Section:Dataset] had an average overlap rate of $11 \%$ between followers of two influencers. As the number of selected 'similar' influencers increased, this overlap rate increased making it an even bigger issue in terms of increasing unique reach. To as certain this problem's magnitude, we considered the following example:

A marketing agency selects four influencers (IA, IB, IC, ID) registered with them, but their total in-house database could be in the millions. The dynamics of these influencers are illustrated in Tables 1 showing the followers, Table 2 tallying the true reach and Figure 1 which shows the graphical representation. 
Table 1: Sample Four Influencers and their followers

\begin{tabular}{|l|l|l|}
\hline \multicolumn{1}{|c|}{ Influencer } & Total Followers & Followed By \\
\hline IA & 6 & $\mathrm{a}, \mathrm{b}, \mathrm{c}, \mathrm{d}, \mathrm{e}, \mathrm{f}$ \\
\hline IB & 4 & $\mathrm{a}, \mathrm{b}, \mathrm{g}, \mathrm{h}$ \\
\hline IC & 4 & $\mathrm{c}, \mathrm{d}, \mathrm{g}, \mathrm{h}$ \\
\hline ID & 3 & $\mathrm{a}, \mathrm{i}, \mathrm{j}$ \\
\hline
\end{tabular}

Table 2: True reach for each possible follower combination

\begin{tabular}{|l|l|l|}
\hline Selected Influencer & Sum of Followers & True Reach \\
\hline IA & 6 & 6 \\
\hline IB & 4 & 4 \\
\hline IC & 4 & 4 \\
\hline ID & 3 & 3 \\
\hline IA, IB & 10 & 8 \\
\hline IA, IC & 10 & 8 \\
\hline IA, ID & 9 & 8 \\
\hline IB, IC & 8 & 6 \\
\hline IB, ID & 7 & 6 \\
\hline IC, ID & 7 & 7 \\
\hline IA, IB, IC & 14 & 8 \\
\hline IA, IB, ID & 13 & 10 \\
\hline IB, IC, ID & 11 & 8 \\
\hline IA,IB,IC,ID & 17 & 10 \\
\hline
\end{tabular}

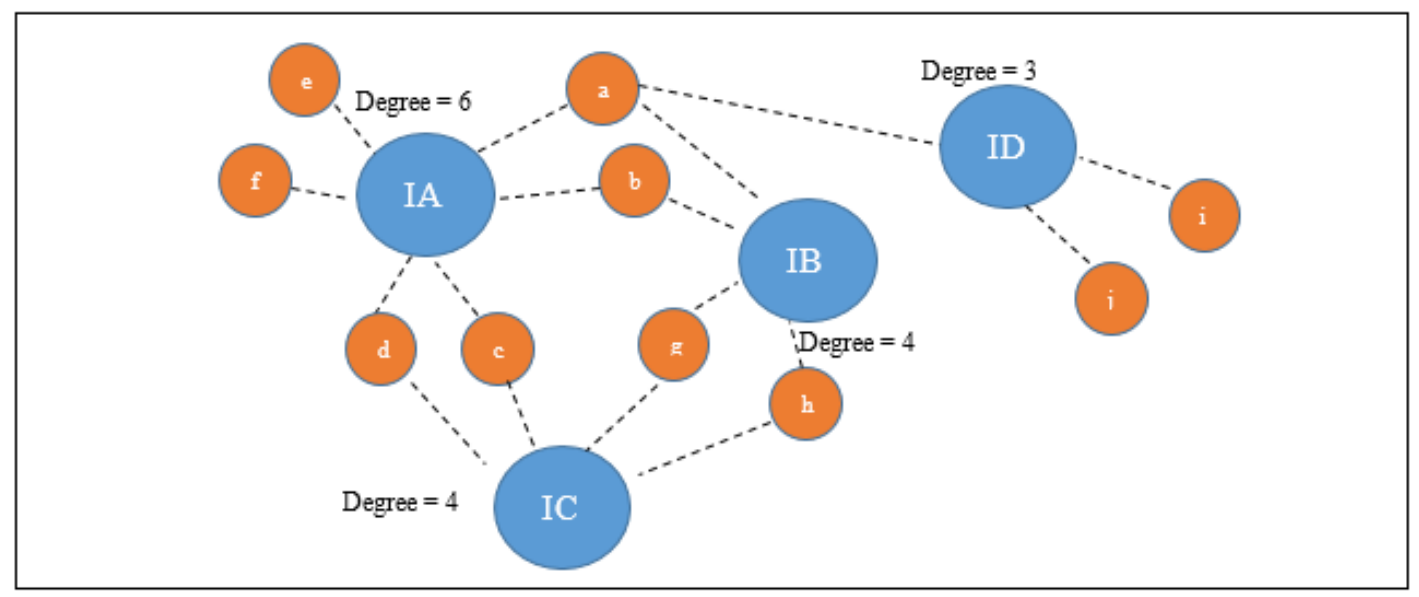

Figure 1 - Graphical data representation.

Larger nodes represent influencer-nodes while smaller nodes represent follower-nodes.

From the above scenario, influencer IC's followers can all be reached with influencers IA and IB so the addition of influencer IC does not increase the total reach. The impact of a brand's selection constrained by budget if they limit by having:

(1) Two influencers: choosing IA and ID will be more economical over the obvious choice of IA-IB or IA-IC, as the true reach of IA-ID is 8 which is the same as the true reach of IA- 
IB and IA-IC and more than the true reach of IB-IC that is 6, as ID has less number of followers than IB and IC and should charge less than the other two (Ref Figure 1 and Table 2), an improved reach by $33 \%$.

(2) Three influencers: the combination of IA-IB-ID will be more efficient than IA-IB-IC despite the sum of total followers of IA-IB-IC (14) being more than the total followers of IA-IB-ID(13) as the true reach of IA-IB-ID is 10 while that of IA-IB-IC is 8 , an improved reach of $25 \%$.

In the real world, the graph of all the available influencers and their followers, contains millions of nodes and edges so to find the best solution, one will have to explore all the possible combinations resulting in the complexity of $\mathrm{O}\left(2^{\mathrm{n}}\right)$.

\section{OUR Solution}

To solve the problem of reaching the maximum audience by selecting specific influencers from a set of influencers, we propose a Greedy Algorithm. The heuristic is to keep combining pair of influencers from a set of available influencers that generates the maximum reach until the required reach or set of influencers is obtained. We used SNAP-Stanford Network Analysis Project[11] to generate and manipulate the graph.

\subsection{Parameters}

The proposed algorithm allows users to set following parameters, or alternatively the algorithm can select a default value.

\subsubsection{Target Reach}

Total audience the brand wants to target. The set of influencers under consideration should have total number of unique followers equal to or greater than the targeted reach.

\subsubsection{Macro Influencer Threshold}

Minimum number of followers required by the influencer to be considered a Macro Influencer. The system uses a default value of 300,000 .

\subsubsection{Micro Influencer Threshold}

Minimum number of followers required by the influencer to be considered a Micro Influencer. The system uses a default value of value is 100,000. Any influencer having followers between Micro Influencer Threshold and Micro Influencer Threshold is considered Micro Influencer.

\subsubsection{Number of Macro Influencers}

The maximum number of macro influencers who can be part of selected influencers.

\subsubsection{Number of Micro Influencers}

The maximum number of micro influencers who can be part of selected influencers. 


\subsection{Methodology}

The goal is to manipulate the influencer graph so that the sum of degrees of the influencer nodes produces the true reach of the influencers. To obtain the true reach of two influencers, a two-step process was repeated until two nodes are selected:

(1) After selecting the first influencer node, we removed all the nodes representing followers (follower nodes) of the selected influencer from the graph.

(2) The degree of all the influencer-nodes that were connected with the removed follower nodes will decrease ensuring that overlapping follower nodes between the selected influencers are considered only once. (Ref: Figure 2(a) and Figure 2(b))

Note, before removing the nodes from the graph, the degree of the influencer under consideration is stored for future processing.

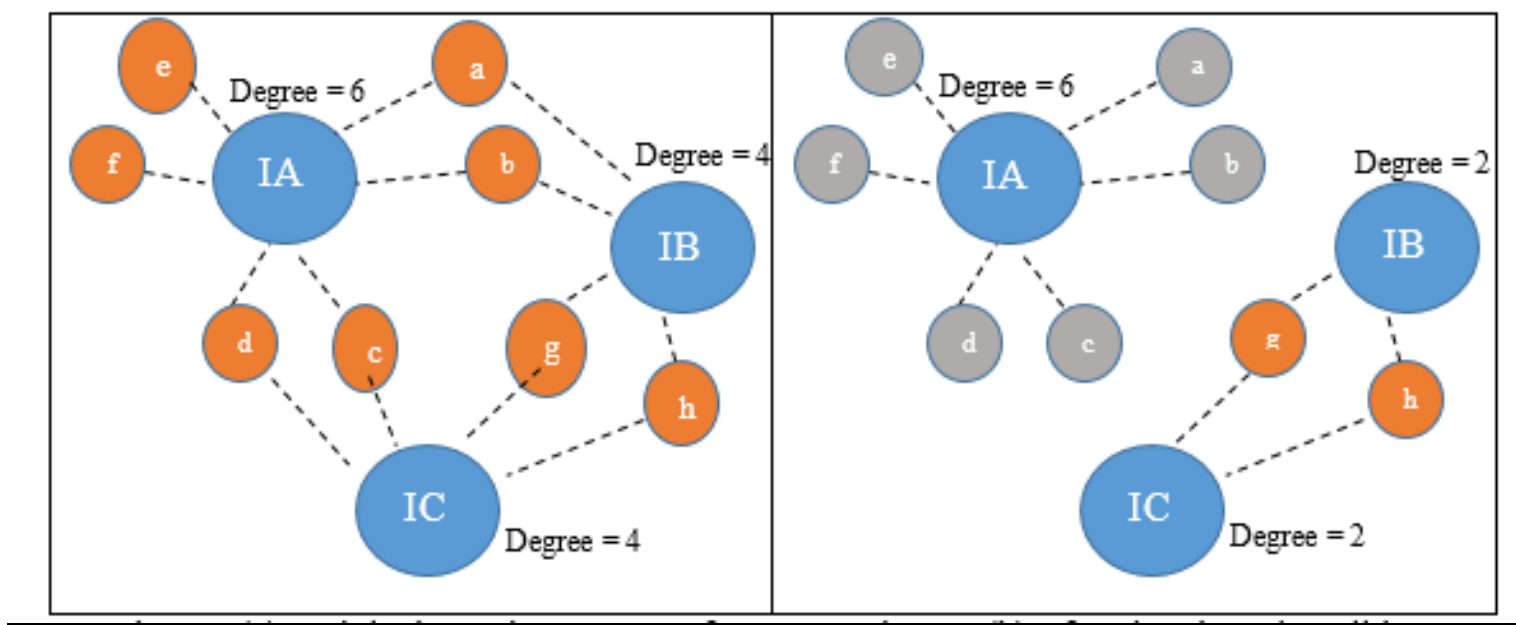

Figure 2(a): Original Graph - Degree of IA:6, IB: 4, IC: 4

Figure 2(b): If IA is selected - All its follower nodes will be removed from the graph, reducing the degree of IB and IC to 2 from 4.

With reference to the example in Figure 2, the influencer IA degree that is 6 is stored and a, $\mathrm{b}, \mathrm{c}, \mathrm{d}, \mathrm{e}, \mathrm{f}$ are removed from the graph. After removing the nodes, the sum of degree of influencer IA and any other influencer will result in the true reach of the both influencers, as the overlapping nodes are only considered while storing the degree of influencer IA.

\subsection{Algorithm}

The first phase of our algorithm is to pre-process and transform the data into a structure that can generate a graph. We create an Influencer dictionary as keys and a complete list of all their followers as the value.

For the example in Figure 2A, our follower Map will be:

$$
\text { followerMap }=\left\{{ }^{\prime} I A^{\prime}:[a, b, c, d, e, f], ' I B^{\prime}:[a, b, g, h], ' I C^{\prime}:[c, d, g, h]\right\}
$$

Once we have the follower Map an undirected graph as represented in Figure(1) is created, using SNAP. 
International Journal of Data Mining \& Knowledge Management Process (IJDKP) Vol.9, No.4, July 2019

\begin{tabular}{|c|c|}
\hline Code & Description \\
\hline 1. completeGraph & 1. Creates undirected graph \\
\hline snap.TUNGraph.New0 & 2. Visits all influencers in our mapping \\
\hline 2. for $\mathrm{i}$ in followersMap: & 3. Checks if a node representing \\
\hline 3. if not completeGraph.IsNode(i): & $\begin{array}{l}\text { influencer exists in graph or not } \\
\text { 4. Adds a new node }\end{array}$ \\
\hline 4. completeGraph.AddNode(i) & 5. Visits all followers of an influencer \\
\hline 5. for $\mathrm{j}$ in followersMap $[\mathrm{i}]$ : & 6. Checks if a node representing \\
\hline 6. if not completeGraph.IsNode(j): & $\begin{array}{l}\text { follower already exists in graph. } \\
\text { 7. Adds a new node for follower }\end{array}$ \\
\hline 7. completeGraph.AddNode(j) & 8. Adds an edge between Influencer and \\
\hline 8. completeGraph.AddEdge(i,j) & follower \\
\hline 9. FOut & $\begin{array}{l}\text { 9-11.Saves the initial state of the graph for } \\
\text { later use. }\end{array}$ \\
\hline 10. completeGraph.Save(FOut) & \\
\hline 11. FOut.Flush & \\
\hline
\end{tabular}

The main algorithm is summarized in the following steps:

(1) Select an influencer node (i)

(2) Store the degree of influencer node (i) and remove all its follower nodes (followers) from the main graph, degrees of all the influencer nodes sharing the removed follower nodes will be updated

(3) Visit every other influencer node and add the degree of each influencer node in the degree of influencer node (i) and find the pair that results in highest reach, i.e., has the highest sum of degrees. Record the value of this reach and the selected pair.

(4) Restore the graph's initial state and perform steps 1 to 3 for each influencer node.

(5) Select the pair that results in highest reach. The reach is stored, and all their follower nodes are removed from the graph. The graph's initial state is updated with this state, i.e., after the removal of follower nodes of selected influencer nodes.

(6) Repeat steps 3 to 5 until the targeted reach is achieved or all influencer nodes have been selected.

\subsubsection{Finding the first influencer pair}

After creating the graph in Figure 1, the first step is to find the initial two influencer nodes that will result in highest reach is shown in Figure 3a.

\begin{tabular}{|c|c|}
\hline Code & Description \\
\hline 1. shortlistedInfluencers $=[]$ & 1. Defines List to store the selected \\
\hline 3. for $\mathrm{i}$ in followersMap: & 2. Defines List to record the visited \\
\hline 4. FIn = snap.TFIn("completeGraph.graph") & influencer \\
\hline $\begin{array}{l}\text { 5. completeGraph = snap.TUNGraph.Load(FIn) } \\
\text { 6. } \text { audienceA = completeGraph.GetNI(i).GetDeg() }\end{array}$ & $\begin{array}{l}\text { 3. Visits all influencer nodes in our } \\
\text { graph (i) }\end{array}$ \\
\hline n followersMap $[\mathrm{i}$ & 4-5 Restores previous state of the graph \\
\hline $\mathrm{n}$ follower & node \\
\hline elNode(node) & under \\
\hline 10. visited & 7-9. Removes all follower nodes of the \\
\hline for $\mathrm{j}$ & the graph \\
\hline if $\mathrm{j}$ in visitedNodes: & 10 Marks \\
\hline ze & \\
\hline $\begin{array}{l}\text { audienceSize } \\
\text { audienceA+completeGraph.G }\end{array}$ & $\begin{array}{l}\text { 11-18 Visits all other influencers } \\
\text { checks which influencer cor }\end{array}$ \\
\hline
\end{tabular}


International Journal of Data Mining \& Knowledge Management Process (IJDKP) Vol.9, No.4, July 2019

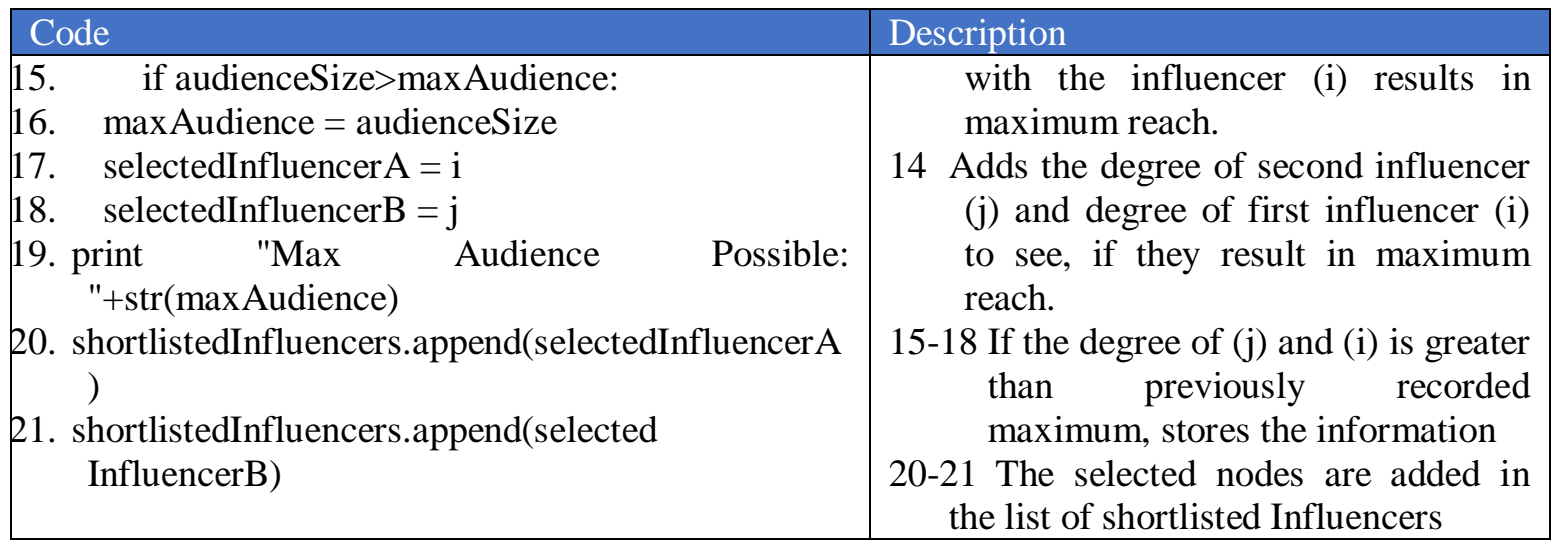

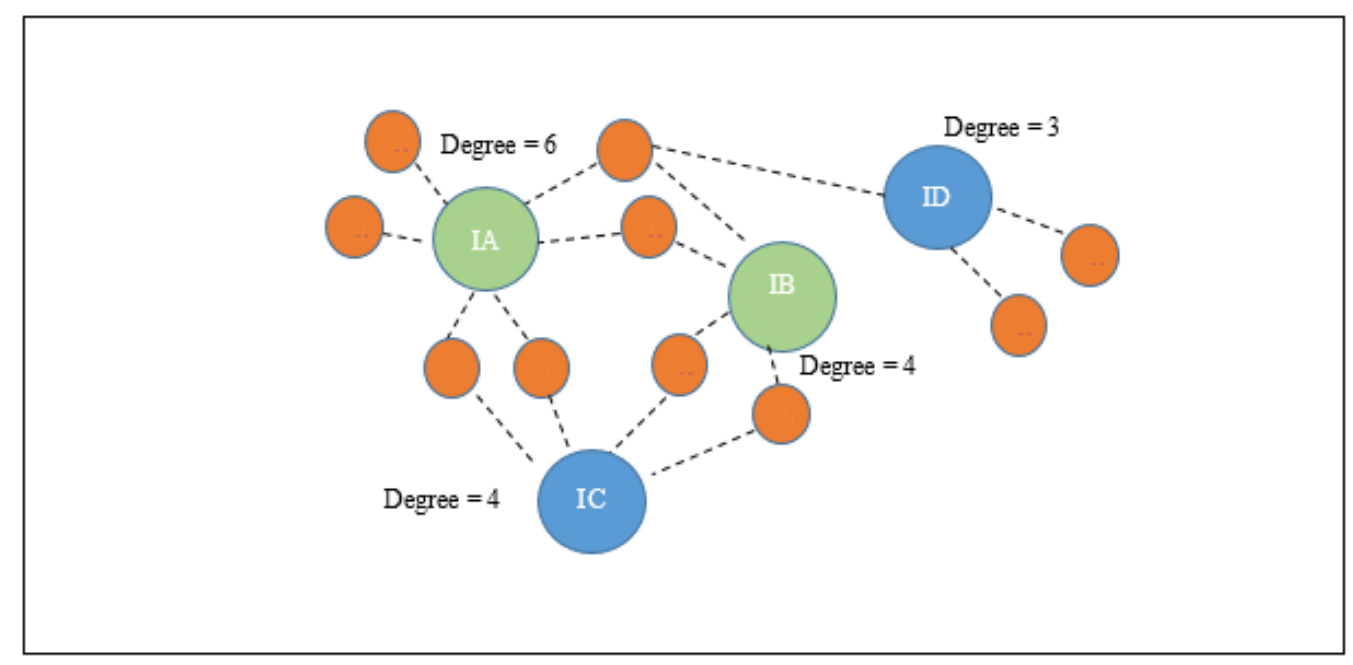

Figure 3(a) - As influencers IA and IB result in the highest reach, they are selected.

\subsubsection{Update Graph States after Initial Influencer Selection}

Once the initial nodes are selected, the sum of their degrees is stored as max Audience, the maximum Audience that can be reached after selection of these two influencer nodes. All the follower nodes are removed from the graph and the graph's initial state is updated to this new state as shown in Figure 3(b).

\begin{tabular}{|c|c|}
\hline Code & Description \\
\hline 1 for $\mathrm{i}$ in shortlisted Influencers: & 1 Visits both shortlisted influencers(i) \\
\hline for node in followers Map[i]: & do $2-5$ \\
\hline $\begin{array}{l}\text { if complete Graph. Is Node(node) and } \\
\text { node not in followers Map: }\end{array}$ & $\begin{array}{l}2 \text { Visits each neighboring node of } \\
\text { shortlisted influencer(i) }\end{array}$ \\
\hline complete Graph. Del Node(node) & $3-4$ Checks if the neighboring node is \\
\hline 5 del followers Map[i] & not influencer, then remove from \\
\hline 6 FOut = snap.TFOut("complete Graph. graph") & graph. \\
\hline 7 complete Graph. Save(FOut) & 5 Removes the shortlisted influencer \\
\hline 8 FOut. Flush() & from the followers Map too as it has \\
\hline & already been shortlisted as a \\
\hline & $\begin{array}{l}\text { 6-8 Update the initial state of the graph } \\
\text { is this modified state. }\end{array}$ \\
\hline
\end{tabular}




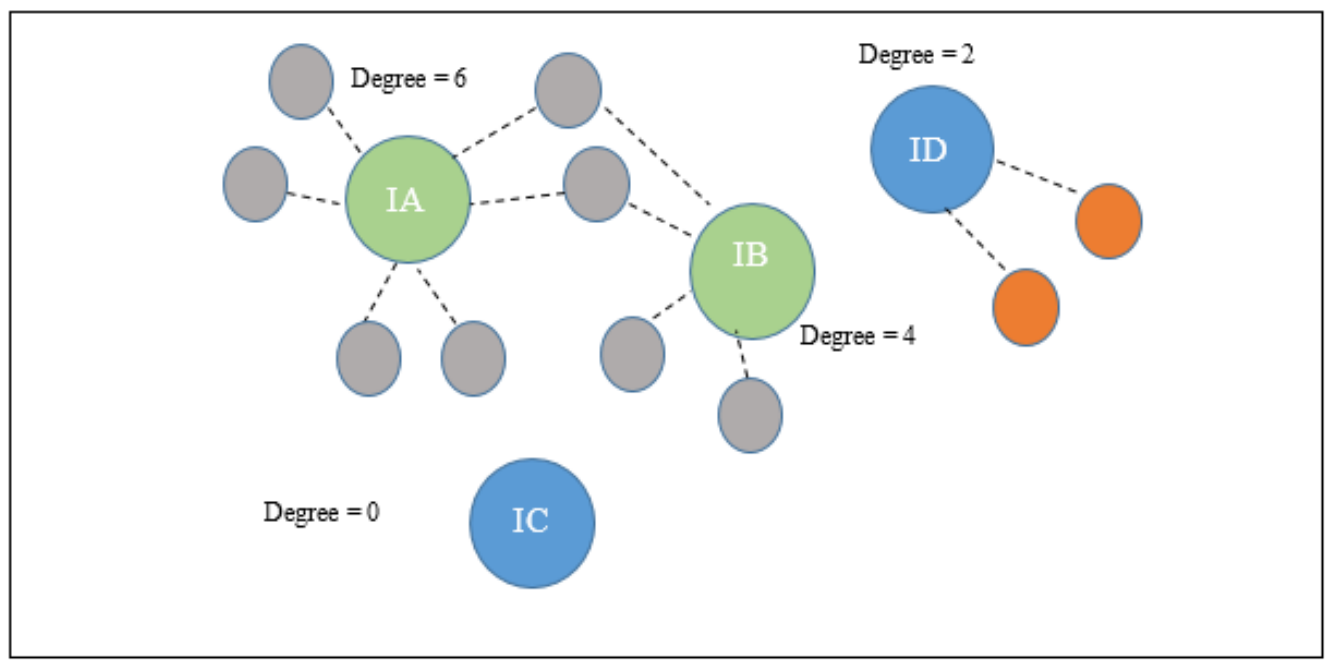

Figure 3(b) - As influencers IA and IB result in the highest reach, they are selected

\subsubsection{Iteratively selecting influencers to achieve desired reach}

Once the initial pair of influencers is selected, the merged pair is compared with every other remaining influencer to measure which of the remaining influencer, if merged with selected influencers, will result in maximum reach. If such an influencer is found, it is merged with already merged influencers. The process continues until the desired reach is achieved or all influencers have been visited, as shown in Figure 3(c).

\begin{tabular}{|c|c|c|}
\hline \multicolumn{2}{|c|}{ Code } & Description \\
\hline & vhile len(followersMap) $>0$ : & 1 Continues until all \\
\hline & current Audience $=$ maxAudience & influencers \\
\hline & if currentAudience>desiredReach: & been explored \\
\hline & break & 2 Stores the audience \\
\hline 5 & audience $=0$ & that can be reached \\
\hline 6 & for $\mathrm{i}$ in followersMap: & with current set of \\
\hline 7 & FIn = snap.TFIn("completeGraph.graph") & influencers \\
\hline & completeGraph = snap.TUNGraph.Load(FIn) & 3-4 Checks if desired \\
\hline , & audience $=$ completeGraph.GetNI(i).GetDeg() & reach \\
\hline 10 & audienceSize $=$ currentAudience+audience & achieved \\
\hline 11 & if not audienceSize<maxAudience: & current set. \\
\hline 12 & max Audience $=$ audienceSize & Audience - to store \\
\hline 13 & selectedInfluencer $=\mathrm{i}$ & followers of the \\
\hline 14 & shortlistedInfluencers.append(selectedInfluencer) & influencer that will \\
\hline 15 & for node in followersMap[selectedInfluencer]: & be explored \\
\hline 16 & $\begin{array}{l}\text { if completeGraph.IsNode(node) and node not in } \\
\text { followersMap: }\end{array}$ & 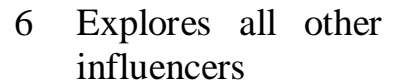 \\
\hline 17 & completeGraph.DelNode(node) & 7-8 Restores the state \\
\hline 18 & del followersMap[selectedInfluencer] & of graph \\
\hline 19 & FOut = snap.TFOut("completeGraph.graph") & 9 Gets the audience \\
\hline 20 & completeGraph.Save(FOut) & that is \\
\hline & FOut.Flush() & being explored \\
\hline & & $\begin{array}{l}10 \text { Getsthe audience } \\
\text { size that can be } \\
\text { reached by adding }\end{array}$ \\
\hline
\end{tabular}



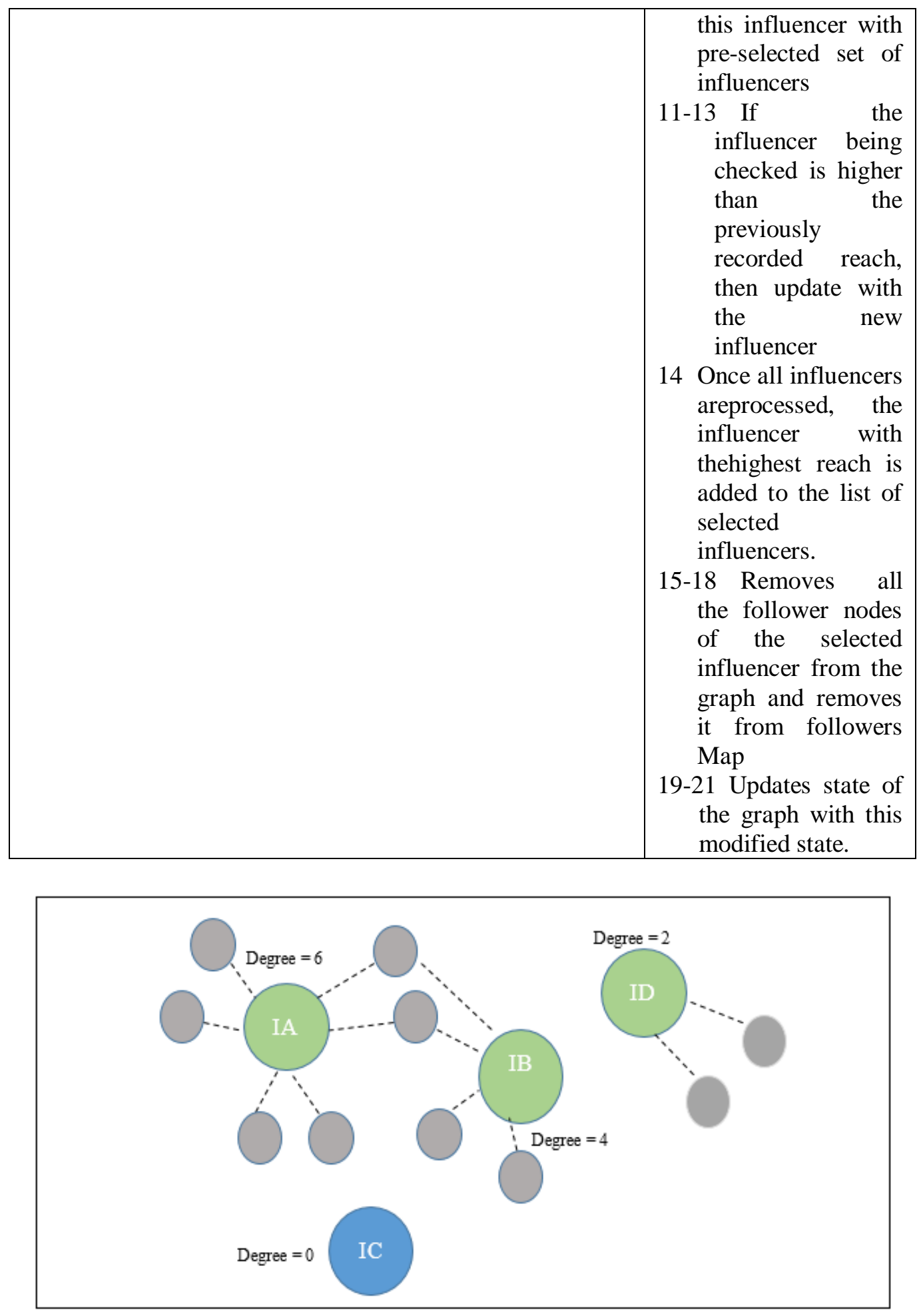

Figure 3(c) - influencer ID combined with influencers IA and IB results in a higher reach than influencer IC combined with IA and IB

Once the script is completed, the variable shortlisted Influencers will have the complete list of all the shortlisted influencers. This general algorithm, run within the script, is further updated to cater for useful parameters, for example: 
(1) The number of followers and influencer should have to be categorized as either a macro-or micro-influencer.

(2) The total number of macro- and micro-influencers who can be part of the final list of shortlisted influencers.

These parameters are important to optimize the results as per the brand's budget. View the complete python script at: bit.ly/2X9lalS

\subsection{Implementation with High Performance Computing}

In order to make our proposed algorithm computational feasible, High Performance Computing (HPC) environment can be employed. We designed a complete pipeline using Nifi to ingest data from our database into Hadoop and calculate the reach of all influencer pairs that is needed at each iteration in parallel. Based on the results of these parallel computations, the influencer is selected that results in highest reach when combined with set of selected influencers see Figure (4).

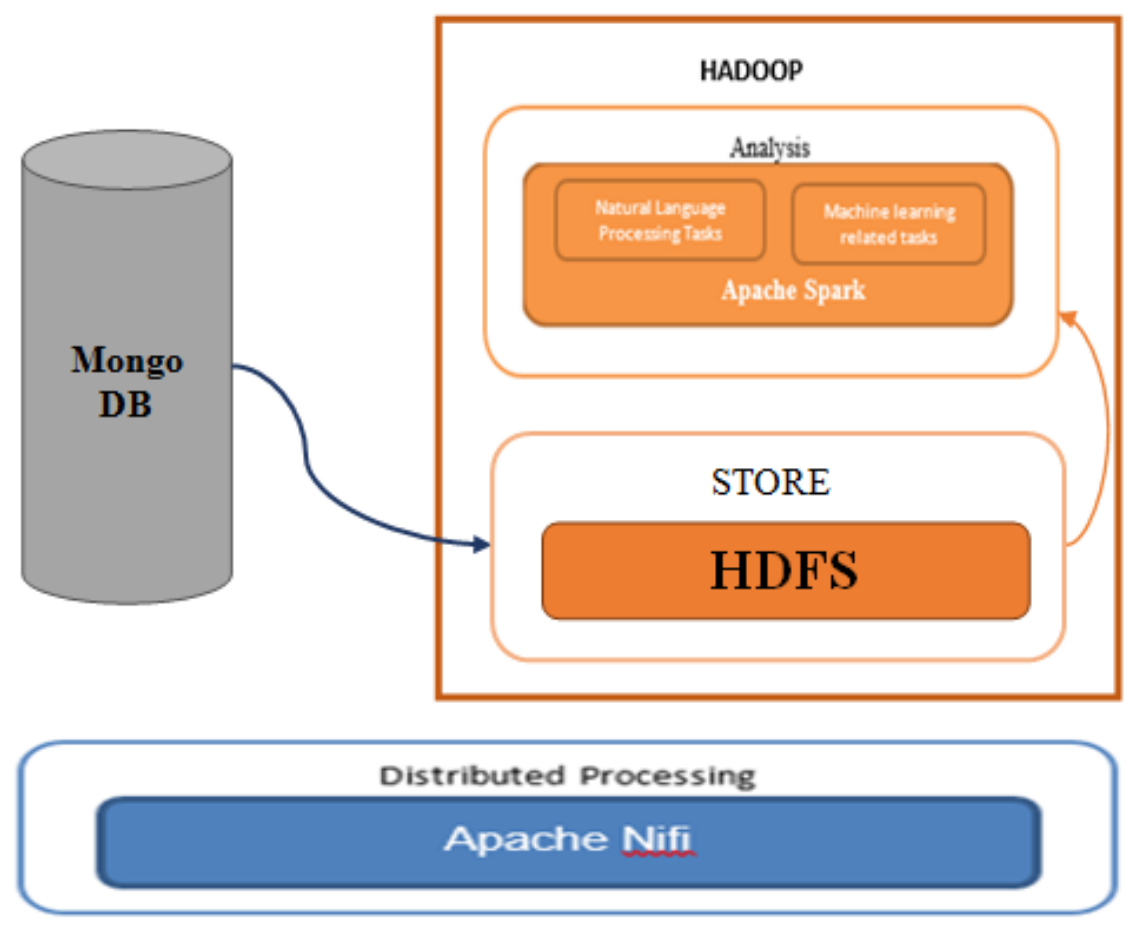

\subsection{Dataset}

Figure 4 - High level architecture of HPC

We identified 500+ influencers from Pakistan with 5,000 to 300,000 followers and collected their publicly available data which includes:

(1) Public profiles including biography, number of followers and number of people they follow

(2) Publicly available profile feeds for the last six months, including the caption of the posts and number of likes and comments received on each post

(3) Followers list. 
To keep our analysis generic and unbiased, we tried to select influencers from a wide variety of thematic areas and niches but despite best efforts, around $70 \%$ of our influencer are fashion bloggers.

\section{RESULTS}

Our objective was to measure the effectiveness of our greedy approach by analyzing the reach and overlap of the influencers selected by our algorithm. We were also interested in establishing the KPIs and benchmarks to measure the performance of Instagram Influencers. This section discusses all the results and observations that were made.

\subsection{Followers Overlap}

The average followers overlap between any two influencers in our dataset was around $11 \%$. The highest overlap between two influencers was found to be $90 \%$ with followers 210,241 and 37,858 . The lowest overlap in our dataset was $0.05 \%$ between two influencers with followers 86,485 and 11,630 .

We used our proposed solution to find the set of influencers that can reach at least 1 million users from our dataset. We also set the constraint of having only 1 influencer having followers more than 200,000 in the final selected set. If our solution wasn't used and top influencers were chosen from the dataset the desired reach could have received with 5 influencers having followers $303060,194519,190430,190343$ and 186984 respectively. In reality the true reach of these 5 followers is only724,302 instead of $\mathbf{1 , 0 6 5 , 3 3 6}$ (sum of reach of all influencers).

Our solution selected 6 influencers with followers 279065, 194945, 190343, 186984, 133920, 131886,101426 respectively. The true reach of selected influencers is $\mathbf{1 , 0 3 5 , 4 7 4}$ and the reach is $\mathbf{1 , 2 1 8 , 5 6 8}$ (sum of reach of all influencers) [Table 3]. It should also be noted that the influencer with highest followers in our dataset having followers 303,060 was not selected by our proposed solution as it has quite a high overlap with the followers of other influencers in our dataset.

\begin{tabular}{|l|l|l|l|}
\hline Method & $\begin{array}{l}\text { Reach of Selected } \\
\text { Influencers }\end{array}$ & Followers Overlap & $\begin{array}{l}\text { True Reach of Selected } \\
\text { Influencers }\end{array}$ \\
\hline $\begin{array}{l}\text { Selecting top } \\
\text { influencers }\end{array}$ & $1,065,762$ & 341,460 & 724,302 \\
\hline $\begin{array}{l}\text { Using Proposed } \\
\text { Greedy Approach }\end{array}$ & $1,218,568$ & 183,094 & $1,035,474$ \\
\hline
\end{tabular}

Table 4 - Method is the technique used to select influencers, Reach of Selected Influencers is the sum of reach of all influencers, Followers Overlap is the number of users following more than one influencer and True reach of Selected Influencers is the number of actual unique users that can be reached with selected influencers.

\subsection{Analysis of Influencer Profiles}

We performed a detailed analysis of our dataset, with the objective to observe a relationship between influencers and their profile activity by analyzing the content they posted and establish benchmarks used to measure the influencers' effectiveness. 


\subsubsection{Average Engagement of a Post}

The average influencers engagement was $3.28 \%$ with only 29 out of 550 influencers had average engagement more than $10 \%$, based on the influencers' profile feed.

Average Engagement $=\frac{\text { Engagement on post }}{\text { Total followers of the influencer }} * 100$

\subsubsection{Post Frequency/Week}

We calculated that most influencers do not post more than three posts in a week and of 550 influencers, about $25 \%$ or 140 posted more than five times/week i.e., more than one post daily as shown in Figure 5.

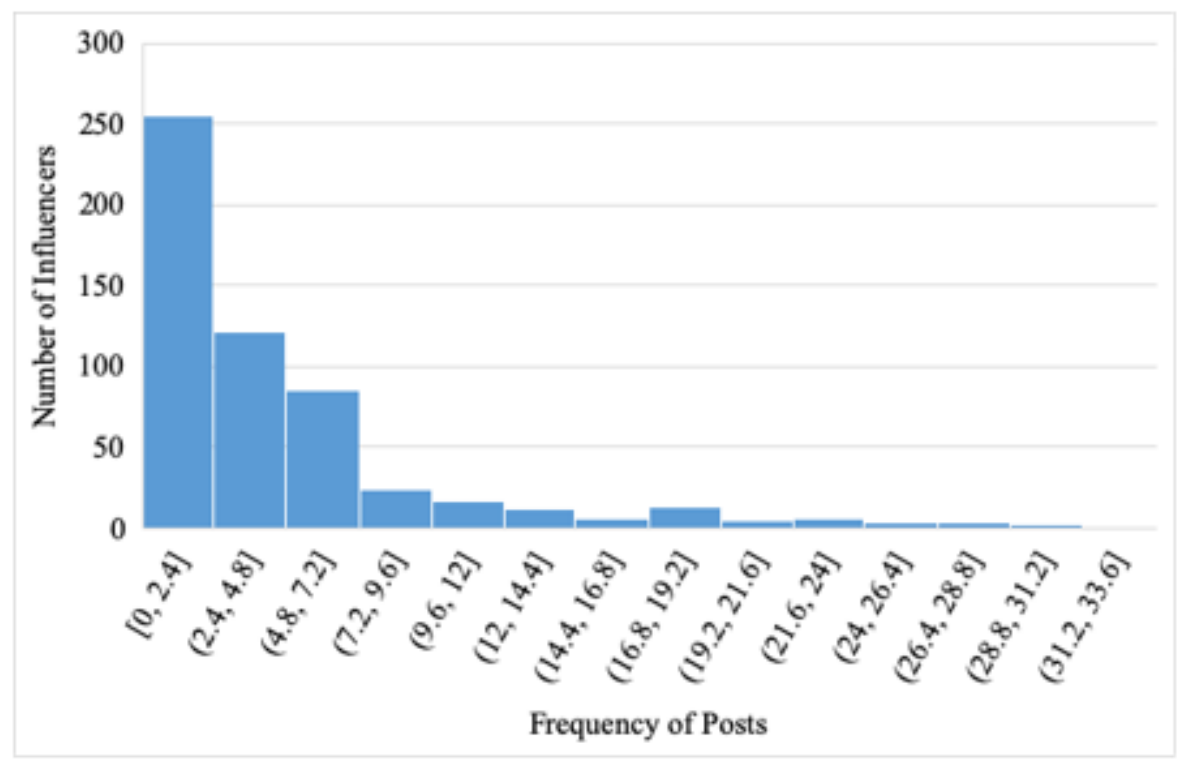

Figure 5 - Post frequency of Influencers

\subsubsection{Average Engagement vs. Post Frequency}

To find the optimum number of weekly posts that an influencer should make, we calculated the average frequency that resulted with the highest engagement number. There was no conclusive trend, but the data showed that the probability of a higher engagement is with less number of weekly posts i.e., influencers who posted 2 to 3 times/ week received relatively better engagement rates than influencers who posted more frequently as shown in Figure 6. 
International Journal of Data Mining \& Knowledge Management Process (IJDKP) Vol.9, No.4, July 2019

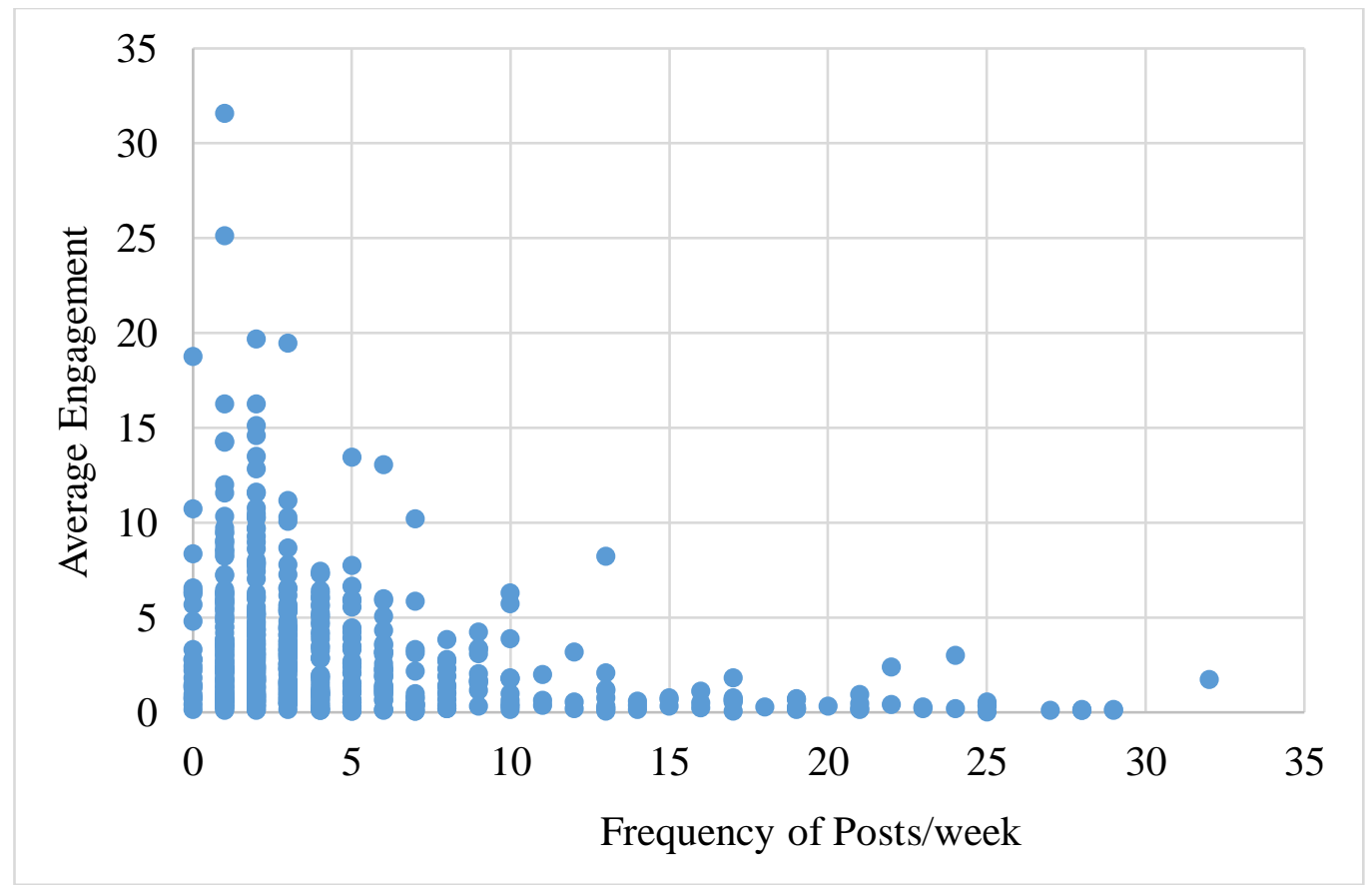

Figure 6-Average Engagement vs. Frequency of Posting/week

\subsubsection{Average Engagement vs. Caption Length}

Posts with longer captions are more likely to get higher engagement than the posts with shorter caption based on analyzing 100,000+ posts by550 influencers. Around 35\% of the posts analyzed had caption length less than 200 characters, around $45 \%$ had lengths between 200 and 600 characters and only $20 \%$ had caption length of more than 600 characters, as illustrated in Figure 7.

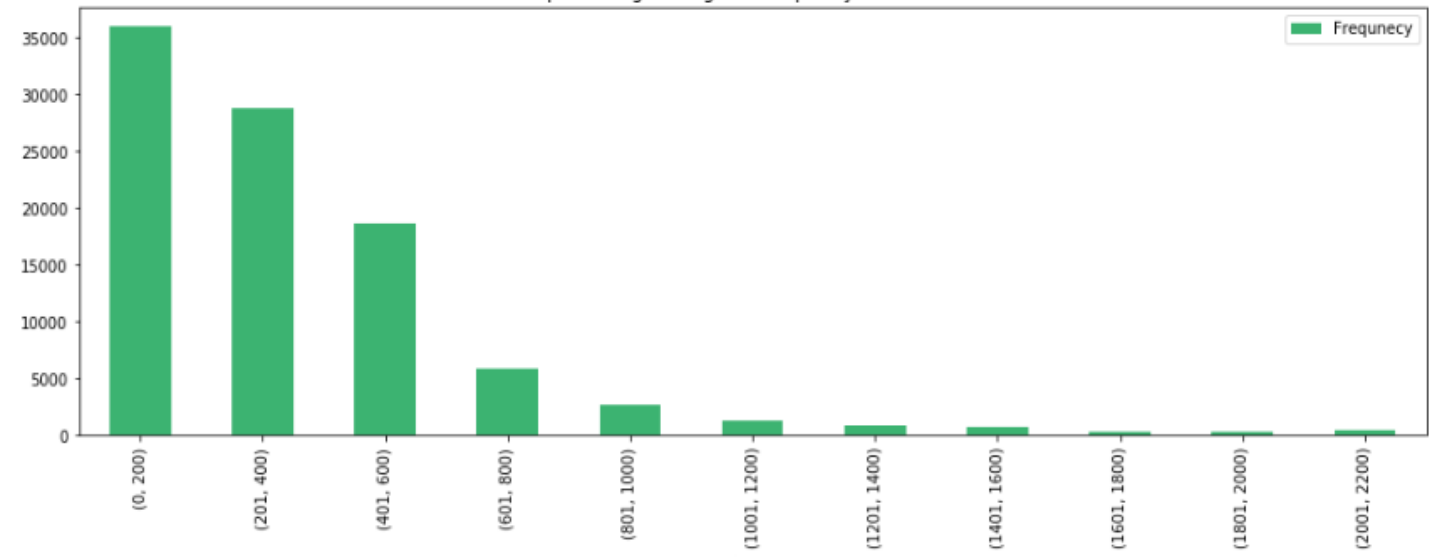

Figure 7: Caption Length Range vs. Frequency of Occurrence

The analysis shows posts with longer captions are more likely to get good engagement. Based on the analysis, every post with higher than the $5 \%$ engagement rate has a caption length longer than 500 characters, as shown in Figure 8. 


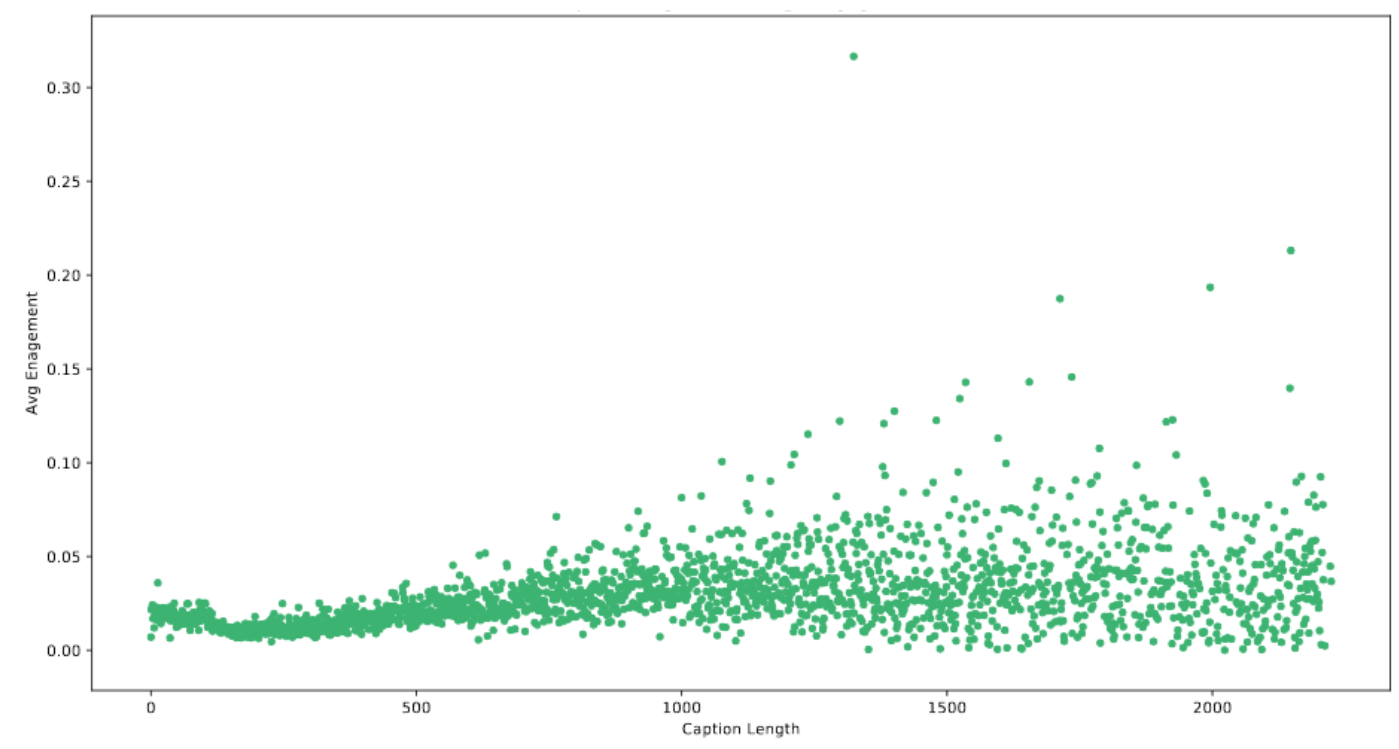

Figure 8: Caption Length vs. Average Engagement

\section{Constraints And Limitation}

The key constraint to our approach is to have a comprehensive list of followers of all the influencers as overlap cannot be found between the influencers without the complete list of all the users following the influencers. In our case, Instagram does not support any method to automatically download or acquire the list of all the followers, so, it becomes an additional cumbersome task to obtain the complete follower lists of all the influencers registered with the agency. In order to remain up to date, these lists need to be updated rather frequently too.

\section{FUTURE WORK}

During this work, we proposed the Greedy Algorithm approach to calculate the optimal set of influencers to maximize reach. This work can be extended to find the optimal set of influencers that maximizes impressions, without considering reach, thereby enabling brands to reach the same audience set multiple times via different influencers. In generating the optimal influencer set, users could only restrict the number of macro- and micro-influencers. To better optimize brands' budgets, the influencers sets can be further processed to include influencers' compensation rates.

\section{ConClusion}

Our main goal was to propose a Greedy Algorithm for marketing agencies performing influencer marketing, to choose an optimal set of influencers to achieve higher reach by analyzing all the possible sets of their registered/accessible influencers. We showed the problem's complexity to find the best solution i.e., the influencers sub-set who are likely to generate the maximum reach as $\mathrm{O}\left(2^{\mathrm{n}}\right)$. Our proposed solution finds the optimal solution in $\mathrm{O}\left(\mathrm{n}^{2}\right)$.

Further, after analysis of 500+Instagram influencers and 100,000+ posts over six months, we found the average influencer engagement rate is $3.28 \%$ of their followers. The data also showed that quality content was more important than quantity to achieve higher engagement rates as influencers who posted up to three times per week and posts with caption lengthsof 500+ characters had higher engagement numbers. 


\section{ACKNOWLEDGEMENTS}

The work presented is part of project "Big Data Analytics \& Artificial Intelligence for Consumer Profiling in Pakistan" fully funded by Ignite (formerly National ICT R\&D Fund). We would also like to extend our gratitude to anonymous reviewers for their valuable insights and recommendations.

\section{REFERENCES}

[1] Gallup \& Gilani Pakistan, " $48 \%$ internet users in Pakistan claim to use social networking sites to access news at least once a day", Gallup Pakistan, https://gallup.com.pk/48-internet-users-in-pakistanclaim-to-use-social-networking-sites-to-access-news-at-least-once-a-day/, Accessed: 6-June-2019

[2] Pakistan Telecommunication Authority, "Telecom Indicators", Pakistan Telecommunication Authority, https://www.pta.gov.pk/en/telecom-indicators ,Accessed: 6-June-2019

[3] Fahad Khan Niazi, "Policy \& regulatory bottlenecks for digital financial services in pakistan", Karandaaz, $\quad$ https://karandaaz.com.pk/wp-content/uploads/2019/06/Policy-Brief-New-1.pdf ,Accessed: 6-June-2019

[4] Influencer Marketing Hub, "The State of Influencer Marketing 2019 : Benchmark Report", Influencer Marketing Hub, https://influencermarketinghub.com/influencer-marketing-2019-benchmark-report/, Accessed: 6-June-2019

[5] Sammis, K., Lincoln, C., Pomponi, S., Ng, J., Gassman Rodriquez, E., \& Zhou, J. (2015). Influencer Marketing for Dummies. Hoboken, New Jersey: Wiley

[6] Freberg K., Graham K., McGaughey K., Freberg L. (2011). Who are the social media influencers? A study of public perceptions of personality, Public Relations Review.

[7] AvinashBhamikar A., Ramchandra Rao P., (2012). Detecting Cliques Using Degree and Connectivity Constraints, International Journal of Data Mining \& Knowledge Management Process

[8] Leskovec J., J. Lang K., Dasgupta A., Mahoney M. (2011) Community Structure in Large Networks: Natural Cluster Sizes and the Absence of Large Well-Defined Clusters, International Mathematics.

[9] Leskovec J., Backstrom L., Kleinberg J. (2009), Meme-tracking and the Dynamics of the News Cycle, International Conference on Knowledge Discovery and Data Mining

[10] SawhneyKartik, Presetio Marcella Cindy, PualSuvadip (2013), Community Detection Using Graph Structure and Semantic Understanding of Text, Knowledge-Based Systems

[11] Leskovec J., Rock S. (2016), SNAP: A General-Purpose Network Analysis and Graph-Mining Library, ACM Transactions on Intelligent Systems and Technology (TIST) 
International Journal of Data Mining \& Knowledge Management Process (IJDKP) Vol.9, No.4, July 2019

\section{Authors}

Salman Ahmad Ansari Working as a Data Scientist in Techlets Pvt Ltd., Pakistan. Area of Interests: Machine Learning, Graph Theory, Data Analysis, Social Media Marketing and Natural Language Processing.

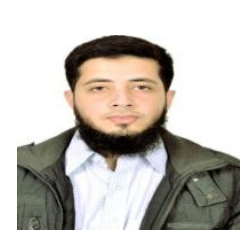

Ahsan Tahir CEO at Techlets Pvt Ltd, Pakistan. Vast Experience in Big Data Analytics

Syed Shahwaiz Bukhari Data Associate at Techlets Pvt Ltd., Pakistan. Area of Interests: Big Data Analytics, Deep Learning and Data Analysis. 